\title{
Approximate Solution for Nonlinear Oscillation of a Mass Attached to a Stretched Elastic Wire by Optimal Homotopy Asymptotic Method
}

\author{
M. Khalid \\ Department of Mathematical Sciences \\ Federal Urdu University of Arts, Sciences \& Techonology \\ University Road, Karachi-75300, Pakistan \\ Faheem Zaidi \\ Department of Mathematical Sciences \\ Federal Urdu University of Arts, Sciences \& Technology \\ University Road, Karachi-75300, Pakistan
}

\author{
Mariam Sultana \\ Department of Mathematical Sciences \\ Federal Urdu University of Arts, Sciences \& Technology \\ University Road, Karachi-75300, Pakistan \\ Azhar Iqbal \\ Department of Basic Sciences \\ Dawood University of Engineering \& Technology, \\ M.A Jinnah Road, Karachi-74800, Pakistan
}

\begin{abstract}
A precise solution of a mathematical model of a mass connected to an elastic wire is being given in this work. The Optimal Homotopy Asymptotic Method is applied to solve this conventional model. Also, comparison with other numerical methodologies and its exact solution will be given for distinct amplitude of oscillations and compliance can be observed. Results suggest that this technique is useful for solving non-linear oscillatory system quite easily. The solution procedure confirm that this method can be easily extended to other kinds of non-linear oscillators.
\end{abstract}

\section{Keywords}

Optimal Homotopy Asymptotic Method; Non-linear Oscillatory System; Small and Large Amplitude; Highest Degree of Accuracy.

\section{INTRODUCTION}

Consider, a mass connected to a strained elastic wire then the nondimensional equation of its motion be [2].

$$
u^{\prime \prime}(t)+u(t)-\frac{\lambda u(t)}{\sqrt{1-u^{2}(t)}}=0 ; \quad 0<\lambda \leq 1
$$

with $u(0)=A$ and $u^{\prime}(0)=0$. Consider this an example of an irrational elastic item placed in a conservative nonlinear oscillatory system. The vacillation of this system is between $[-A, A]$ i.e. symmetric bounds, and its angular frequency along with its corresponding periodic solution depends upon the amplitude $A$.

This problem has been addressed by many a scientist. Nonetheless, deriving an analytical solution for it in a detailed form seems improbable except in some unique situations, such as [1] by combining Newton's and harmonic balance technique derived the approximated analytical solution for non dimensional equation (1). Ergo, one has to choose numerical methods or approximate approaches to obtain its solution.In [3] a good approximation to
Eq.(1) is shown by using parameter-expansion method prsented by He. [4] calculated another approximate solution of Eq.(1) using VIM and energy balance method. Whereas, [5, 6] used homotopy perturbation and energy balance method to approximate the oscillator (1). Geng [7] studied the behavior of a nonlinear oscillator of a mass connected to a strained elastic wire by Piecewise Variational Iteration Method on a massive region, but [7] analyzed that by using standard Variational Iteration Method (VIM) better approximation can be obtained in a fairly small region.

In [8] a new technique by Marinca and Herisanuin is being introduced i.e. Optimal Homotopy Asymptotic Method (OHAM). Difference between OHAM and HAM is that OHAM is more flexible and has implicit convergence criteria. Several studies [9-17] have proved the efficiency, wide spread application and reliability of this method and have used it to obtain solutions of current important problems in the fields of science and engineering.Work presented in this paper signify that OHAM is the most reliable technique to determine the approximated solution of cases like Eq.(1). In this work, to show the accuracy and efficiency of OHAM some numerical examples have been solved with distinct amplitudes. The error analysis of the example confirms the convergence and stability of this technique.

This paper has four sections. Section 2 is about the basic concept of OHAM. In Section 3, OHAM is applied to the mathematical model of non-linear oscillator of a mass connected to a strained elastic wire. Section 4 contains the conclusion and discussion of paper.

\section{BASIC PRINCIPLES OF OPTIMAL HOMOTOPY ASYMPTOTIC METHOD}

Consider $L_{I N}$ be the linear function operator, $N_{O N}$ be the nonlinear function operator, $C$ be the boundary operator, $f(x)$ be the known function and $u(x)$ be the unknown function then the nonlinear oscillatory differential equation becomes 


$$
L_{I N}(y(x))+f(x)+N_{O N}(y(x))=0 ; \quad C\left(y, \frac{d y}{d x}\right)=0
$$

On applying OHAM Eq.(2) becomes

$$
(1-q)\left[L_{I N}(y(x, g))+f(x)\right]=M(g)\left[L_{I N}(y(x, g))+f(x)+N_{O N}(y(x, g))\right]
$$

and

$$
C\left(y, \frac{d y}{d x}\right)=0
$$

where $g \in[0,1]$ is the implanted parameter, $y(x, g)$ is an unknown function, $M(0)=0$ and $M(g)$ be a non-zero auxiliary function for $g \neq 0$. The solution $y(x, g)$ varies from $y_{\circ}(x)$ to the solution $y(x)$, as $g$ increases from zero to one, also for $g=0, y_{\circ}(x)$ is obtained from Eq.(3)

$$
L_{I N}\left(y_{\circ}(x)\right)+f(x)=0 ; \quad C\left(y_{\circ}, \frac{d y_{\circ}}{d x}\right)=0
$$

Let $M(g)=\sum_{i=1} c_{i} g^{i}$, where $c_{i}$ are constants. Consider the solution of Eq.(3) in the form

$$
y\left(x ; g, c_{i}\right)=y_{\circ}(x)+\sum_{k \geq 1} y_{k}\left(x, c_{i}\right) g^{k} ; \quad i=1,2,3, \ldots
$$

By replacing, Eq.(5) in Eq.(3) and comparing the coefficients of same powers of $g$, the governing equations of $y_{\circ}(x)$ and $y_{k}(x)$ are obtained i.e.

$$
\begin{gathered}
L_{I N}\left(y_{1}(x)\right)=c_{1}\left(N_{O N}\right)_{\circ}\left(y_{\circ}(x)\right) ; \quad C\left(y_{1}, \frac{d y_{1}}{d x}=0\right) \\
L_{I N}\left(y_{k}(x)-y_{k-1}(x)\right)=c_{k}\left(N_{O N}\right)_{\circ}\left(y_{\circ}(x)+\sum_{i=1}^{k-1}\left[L _ { I N } \left(y_{k-i}(x)+\right.\right.\right. \\
\left(N_{O N}\right)_{k-i}\left(y_{\circ}(x), y_{1}(x), \ldots, y_{k-1}(x)\right] ; \\
C\left(y_{k}, \frac{d y_{k}}{d x}\right), k=2,3, \ldots
\end{gathered}
$$

By expanding $N_{O N}\left(y\left(x ; g, c_{i}\right)\right)$, for $i=1,2,3, \ldots$ in the form of series with respect to $g$ becomes $\left(\left(N_{O N}\right)_{m}\left(y_{\circ}(x), y_{1}(x), \ldots, y_{m}(x)\right)\right.$ i.e. the coefficient of $g$;

$N_{O N}\left(y\left(x ; g, c_{i}\right)\right)=\left(N_{O N}\right)_{\circ}\left(y_{\circ}(x)\right)+\sum_{k \geq 1}\left(N_{O N}\right)_{m}\left(y_{\circ}, y_{1}, y_{2}, \ldots, y_{m}\right) g$

As $y\left(x ; g, c_{i}\right)$ obtained in Eq. (5). It has to be enunciate for $k \geq 0$, $y_{k}$ are conducted by linear Eqs. (4) and (6), having linear boundary conditions provided in the original problem, that can be solved quickly. Convergence of Eq. (5) confide in the auxiliary constant $c_{1}, c_{2}, c_{3}, \ldots$. Therefore, if (5) converges at $g=1$, it becomes

$$
y\left(x, c_{i}\right)=y_{\circ}(x)+\sum_{k=1} y_{k}\left(x, c_{i}\right)
$$

In general terms, the approximate solution of Eq. (1) is obtained as
Table 1. Different values of angular frequency and constant for various values of amplitude.

\begin{tabular}{|c|c|c|c|}
\hline$\lambda$ & $A$ & $\Omega$ & $c_{1}$ \\
\hline \multirow{4}{*}{0.1} & 0.1 & 0.948887 & 0.115588 \\
& 1 & 0.961132 & 0.120648 \\
& 10 & 0.993738 & 0.098365 \\
& 100 & 0.999366 & 0.093508 \\
\hline \multirow{4}{*}{0.5} & 0.1 & 0.708427 & 0.210263 \\
& 1 & 0.786663 & 0.253672 \\
& 10 & 0.968277 & 0.108473 \\
& 100 & 0.996826 & 0.094908 \\
\hline \multirow{5}{*}{0.75} & 0.1 & 0.502795 & 0.430277 \\
& 1 & 0.654292 & 0.521535 \\
& 10 & 0.952011 & 0.115744 \\
& 100 & 0.995234 & 0.095798 \\
\hline & 0.1 & 0.231408 & 2.501239 \\
& 1 & 0.524071 & 1.244578 \\
& 10 & 0.93879 & 0.122188 \\
& 100 & 0.993961 & 0.096521 \\
\hline
\end{tabular}

$$
\left.y^{m}\left(x, c_{i}\right)=y_{\circ}(x)\right)+\sum_{i=1}^{m} y_{k}\left(x, c_{i}\right) ; \quad i=1,2,3, . ., m
$$

By putting Eq.(8) into Eq.(2), the following residual is obtained for $i=1,2,3, . ., m$

$$
R_{e s}\left(x, c_{i}\right)=L_{I N}\left(y^{m}\left(x, c_{i}\right)\right)+f(x)+N_{O N}\left(y^{m}\left(x, c_{i}\right)\right)
$$

$y^{m}\left(x, c_{i}\right)$ becomes the exact solution only if $R_{e s}\left(x, c_{i}\right)=0$. Mostly, these type of cases don't show up for non-linear problems but if such case arise, it can $\mathrm{b}$ minimized by least square or Galerkin Method and find values of $c_{1}, c_{2}, c_{3}, \ldots, c_{m}$

$$
I\left(c_{i}\right)=\int_{a_{1}}^{a_{2}} R_{e s}^{2}\left(x, c_{i}\right) d x
$$

$a_{1}$ and $a_{2}$ are given in the considered problem. Unknown constants $c_{1}, c_{2}, c_{3}, \ldots, c_{m}$ can be obtained from the following known constants in conditions

$$
\frac{\partial I}{\partial c_{1}}=\frac{\partial I}{\partial c_{2}}=\ldots=\frac{\partial I}{\partial c_{m}}=0
$$

therefore the approximate solution (of order $m$ ) is well determined in Eq.(8) .

\section{APPLICATION OF OPTIMAL HOMOTOPY ASYMPTOTIC ON NONLINEAR OSCILLATOR METHOD}

Eq.(1) shows the non-dimensional differential equation of a mass connected to a strained elastic wire having irrational elastic term. On considering a scalar time $\tau=\frac{2 \pi t}{T}=\Omega t$, where $\Omega$ is the unknown parameter which represents frequency that will be calculated later in this study. Under the transformation

$$
t=\Omega t ; \quad y(t)=A u(\tau)
$$

Then original Eq. (1) becomes

$$
\Omega^{2} u^{\prime \prime}(\tau)+u(\tau)-\frac{\lambda u(\tau)}{\sqrt{1-A^{2} u^{2}(\tau)}}=0
$$


with initial condition $u(0)=1$ and $u^{\prime}(0)=0$. Here $u^{\prime}$ denotes the derivative of $\mathrm{y}$ with respect to $\tau$. Therefore a family of equations constructed by OHAM as

$$
\begin{gathered}
(1-q) L_{I N}[\phi(\tau, g)]= \\
\left.\frac{\lambda(\tau, g)\left[\Omega^{2} \phi^{\prime \prime}(\tau, g)+\phi(\tau, g)-\right.}{\sqrt{1-A^{2} \phi^{2}(\tau, g)}}\right]=0
\end{gathered}
$$

and the linear operator given as

$$
L_{I N}(\phi(\tau, g))=\left[\frac{\partial^{2} \phi(\tau, g)}{\partial \tau^{2}}+\phi(\tau, g)\right]
$$

Now $\phi(\tau, 0)=u_{\circ}(\tau) ; \phi(\tau, l)=u(\tau)$ are the initial conditions, $y_{\circ}(\tau)$ is the approximated initial value of $y(\tau)$. Therefore, $\phi(\tau, g)$ fluctuates from $u_{\circ}(\tau)$ to $u(\tau)$ as the embedding parameter $g$ increase from $0 \rightarrow 1$. Explicating $\phi(\tau, g)$ in the form of series with respect to the parameter $g$, one can easily obtain $\phi(\tau, g)=u_{\circ}(\tau)+g u_{1}(\tau)+\ldots$. Let the auxiliary function be :

$$
M(\tau, g)=c_{1} g \cos \tau
$$

The zeroth order problem with initial conditions is written as

$$
g^{\circ}: u_{\circ}^{\prime \prime}(\tau)+u_{\circ}(\tau)=0 ; \quad u(0)=1 \text { and } u^{\prime}(0)=0
$$

which has a solution $u_{\circ}(x)=\cos x$. The first order problem is

$$
\begin{gathered}
g^{1}: u_{1}^{\prime \prime}(\tau)+u_{1}(\tau)=c_{1} \cos \tau\left[\Omega^{2} u_{\circ}^{\prime \prime}(\tau)+u_{\circ}(\tau)-\right. \\
\frac{\lambda u_{\circ}(\tau)}{\sqrt{1-A^{2} u_{\circ}^{2}(\tau)}} ; \quad u(0)=0 \text { and } u^{\prime}(0)=0
\end{gathered}
$$

solution of Eq.(18) is

$$
\begin{aligned}
u_{1}\left(x ; \Omega, c_{1}\right)= & \frac{c_{1}}{12 A^{3}}\left[A \left(-6 A\left(\Omega^{2}-1\right)+2 A\left(\Omega^{2}-1\right) \cos 2 x-\right.\right. \\
& \left.3 \sqrt{2} \lambda \sqrt{2+A^{2}(1+\cos 2 x)}\right)+\cos x\left(-4 A^{3}+\right. \\
& 6 \lambda A \sqrt{1+A^{2}}+4 A^{3} \Omega^{2}-\lambda \ln 8-6 \lambda \ln (A+ \\
& \left.\sqrt{1+A^{2}}\right)+6 \lambda \ln (\sqrt{2} A \cos x+ \\
& \left.\sqrt{2+A^{2}(1+\cos 2 x)}\right)-6 \lambda\left(A^{2}-1\right) \sin x( \\
& \left.\left.\tan ^{-1} \frac{\sqrt{2} A \sin x}{\sqrt{2+A^{2}(1+\cos 2 x)}}\right)\right]
\end{aligned}
$$

Now, using Eq. (8), the solution becomes:

$$
u\left(x, \Omega, c_{1}\right)=u_{\circ}(x)+u_{1}\left(x, \Omega, c_{1}\right)
$$

$$
\begin{aligned}
u\left(x ; \Omega, c_{1}\right)= & \cos x+\frac{c_{1}}{12 A^{3}}\left[A \left(-6 A\left(\Omega^{2}-1\right)+2 A\left(\Omega^{2}-1\right) \cos 2 x-\right.\right. \\
& \left.3 \sqrt{2} \lambda \sqrt{2+A^{2}(1+\cos 2 x)}\right)+\cos x\left(-4 A^{3}+\right. \\
& 6 \lambda A \sqrt{1+A^{2}}+4 A^{3} \Omega^{2}-\lambda \ln 8-6 \lambda \ln (A+ \\
& \left.\sqrt{1+A^{2}}\right)+6 \lambda \ln (\sqrt{2} A \cos x+ \\
& \left.\sqrt{2+A^{2}(1+\cos 2 x)}\right)-6 \lambda\left(A^{2}-1\right) \sin x( \\
& \left.\left.\tan ^{-1} \frac{\sqrt{2} A \sin x}{\sqrt{2+A^{2}(1+\cos 2 x)}}\right)\right]
\end{aligned}
$$

The values of $\Omega$ and $c_{1}$ are calculated with the method of least squares, as mentioned in Eqs.(9-11). These values are presented in Table (1) for corresponding values of amplitude $\lambda$.

For comparison purposed, the approximate periods $\left(T_{V I M}, T_{E B M}, T_{O H A M}\right)$ with exact one $\left(T_{E X A C T}\right)$ are tabulated in Table (2) for a large and small value of amplitude $\lambda$.

\section{CONCLUDING REMARKS}

In this work, the approximated solutions obtained through Optimal Homotopy Asymptotic Perturbation Method are in complete agreement with exact solution and other approximated solutions obtained through several numerical methods. Instead of using other techniques, this method can be a better option to solve such nonlinear oscillator problems.

\section{ACKNOWLEDGMENT}

Authors thank the anonymous reviewers for their precious time and positive criticism in advance for the improvement of this paper. The help of Ms Wishaal Khalid in proof reading is also appreciated.

\section{CONFLICT OF INTERESTS}

There is no conflict of interest among authors regarding publication of this work.

\section{REFERENCES}

[1] Sun, W.P., Wu, B.S., Lim, C.W. (2007) Approximate Analytical Solutions for Oscillation of a Mass Attached to a Stretched Elastic Wire, Journal of Sound and Vibration, 300. pp. 1042-1047.

[2] Mickens, R.E. (1996) Oscillations in Planar Dynamics System, World Scientific Publishing, Singapore.

[3] Lan, X. (2007) Application of He's Parameter Expansion Method to an Oscillation of a Mass Attached to a Stretched Elastic Wire, Physics Letter A, 368. pp. 259-262.

[4] Jamshidi, N., Ganji, D.D. (2010) Application of Energy Balance Method and Variational Iteration Method to an Oscillation of a Mass Attached to a Stretched Elastic Wire, Current Applied Physics, 10. pp. 484-486.

[5] Beledez, A., Hernandez, A., Beledez, T., Alvarez, M.L., Gallego, S., Ortuno, M., Neipp, C. (2007) Application of the Harmonic Balance Method to a Nonlinear Oscillator Typified by a Mass Attached to a Stretched Wire, Journal of Sound and Vibration, 302. pp. 1018-1029. 
Table 2. Comparison between OHAM with exact solution and solution obtained by other methods for different values of $\lambda$

\begin{tabular}{|c|c|c|c|c|c|}
\hline$\lambda$ & $A$ & $T_{V I M}[7]$ & $T_{E B M}[4]$ & $T_{O H A M}[$ Our $]$ & $T_{E X A C T}$ \\
\hline & 0.1 & 6.621237 & 6.621737 & 6.62168 & 6.621681 \\
0.1 & 1 & 6517854 & 6.535726 & 6.53727 & 6.537508 \\
& 10 & 6.314678 & 6.320056 & 6.32278 & 6.322938 \\
& 100 & 6.286328 & 6.282868 & 6.28717 & 6.287183 \\
\hline & 0.1 & 8.863794 & 8.869817 & 8.86921 & 8.869257 \\
0.5 & 1 & 7.814722 & 7.972828 & 7.98713 & 7.992133 \\
& 10 & 6.445572 & 6.474308 & 6.48904 & 6.490208 \\
& 100 & 6.298952 & 6.301664 & 6.30319 & 6.303281 \\
\hline & 0.1 & 12.47385 & 12.49906 & 12.4965 & 12.49673 \\
0.75 & 1 & 9.168186 & 9.56407 & 9.60303 & 9.625404 \\
& 10 & 6.531632 & 6.576648 & 6.59991 & 6.602092 \\
& 100 & 6.30688 & 6.31097 & 6.31327 & 6.313403 \\
\hline & 0.1 & 26.86123 & 27.18491 & 27.1524 & 27.15678 \\
0.95 & 1 & 10.96676 & 11.87373 & 11.9892 & 12.07527 \\
& 10 & 6.603018 & 6.662118 & 6.69285 & 6.696116 \\
& 100 & 6.313246 & 6.31844 & 6.32137 & 6.321529 \\
\hline
\end{tabular}

[6] Beledez, A., Beledez, T., Neipp, C., Alvarez, M.L., Hernandez, A. (2009) Approximate Solutions of a Nonlinear Oscillator Typified as a Mass Attached to a Stretched Elastic Wire by the Homotopy Perturbation Method, Chaos Solitons Fractals, 39. pp. 746-764.

[7] Geng, F. (2011) A Piecewise Variational Iteration Method for Treating a Nonlinear Oscillator of a Mass Attached to a Stretched Elastic Wire, Computers and Mathematics with Applications, 62. pp. 1641-1644.

[8] Marinca, V., Herisanu, N. (2008) Application of Homotopy Asymptotic Method for Solving Non-linear Equations Arising in Heat Transfer, International Communications in Heat and Mass Transfer, 35(6). pp. 710-715.

[9] Herisanu, N., Marinca, V. (2010) Accurate Analytical Solutions to Oscillators with Discontinuities and FractionalPower Restoring Force by Means of the Optimal Homotopy Asymptotic Method, Computer and Mathematics with Applications, 60(6). pp. 1607-1615.

[10] Marinca, V., Herisanu, N. (2014) The Optimal Homotopy Asymptotic Method for Solving Blasius Equation, Applied Mathematics and Computation, 231. pp. 134-139.

[11] Iqbal, S., Idrees, M., Siddiqui, A.M., Ansari, A.R. (2010) Some Solutions of the Linear and Nonlinear KleinGordon Equations Using the Optimal Homotopy Asymptotic Method, Applied Mathematics and Computation, 216(10). pp. 2898-2909.
[12] Iqbal, S., Javed, A. (2011) Application of Optimal Homotopy Asymptotic Method for the Analytic Solution of Singular Lane-Emden Type Equation, Applied Mathematics and Computation, 217(9). pp. 7753-7761.

[13] Hashmi, M.S., Khan, N., Iqbal, S. (2012) Optimal Homotopy Asymptotic Method for Solving Nonlinear Fredholm Integral Equations of Second Kind, Applied Mathematics and Computation, 218(22). pp. 10982-10989.

[14] Khan, N., Mahmood, T., Hashmi, M.S. (2013) OHAM Solution for Thin Film Flow of a Third Order Fluid Through Porous Medium over an Inclined Plane, Heat Transfer Research, 44(8). pp. 1-13.

[15] Hashmi, M.S., Khan, N., Mahmood, T. (2013) Optimal Homotopy Asymptotic Solution for Thin Film Flow of a Third Order Fluid with Partial Slip, World Applied Sciences Journal, 21(12). pp. 1782-1788.

[16] Marinca, V., Herisanu, N. (2010) Determination of Periodic Solutions for the Motion of a Particle on a Rotating Parabola by Means of the Optimal Homotopy Asymptotic Method, Journal of Sound and Vibration, 329(6). pp. 14501459.

[17] Khalid, M., Mariam, S., Faheem, Z., Aurangzaib. (2016) A Numerical Solution of Troeschs Problem via Optimal Homotopy Asymptotic Method, International Journal of Computer Applications, 140(5). pp. 1-5. 